\title{
Evaluation of Urine Polymerase Chain Reaction Test Positivity Rates and the Effectiveness of Positron Emission Tomography in Renal Involvement in Patients with Active COVID-19 Infection: A Prospective and Multidisciplinary Study
}

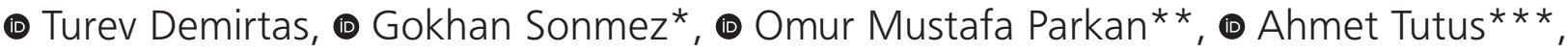 \\ ๑ Zeynep Ture ${ }^{* * *}$, ๑ Sevket Tolga Tombul*, ๑ Orhan Yildiz****, \\ - Selma Gokahmetoglu**, • Emrah Kizilay*, ๑ Abdullah Demirtas* \\ Erciyes University, Medical History and Ethics, Kayseri, Turkey \\ ${ }^{*}$ Erciyes University Faculty of Medicine, Department of Urology, Kayseri, Turkey \\ ${ }^{* *}$ Erciyes University Faculty of Medicine, Department of Medical Microbiology, Kayseri, Turkey \\ $* * *$ Erciyes University Faculty of Medicine, Department of Nuclear Medicine, Kayseri, Turkey \\ $* * * *$ Erciyes University Faculty of Medicine, Department of Infectious Diseases, Kayseri, Turkey
}

\section{Abstract}

\begin{abstract}
Aim: It is known that the Coronavirus disease-2019 (COVID-19) does not only affect the respiratory system in the body, but also affects many vital systems. In this study, we aimed to investigate polymerase chain reaction (PCR) positivity rates in urine samples of patients with COVID-19 infection and to evaluate the effectiveness of positron emission tomography/computed tomography (PET/CT) in demonstrating renal involvement in patients with urinary system involvement findings.
\end{abstract}

Methods: Patients who had positive COVID-19 PCR test and were hospitalized in Erciyes University pandemic wards due to COVID-19 infection between June 2020 and December 2020 were included in this prospective study. A urine PCR test was applied to all patients. In addition, PET/CT was performed in patients with no known malignancy, clean urine culture, but suspected COVID-19 urinary system involvement.

Results: A total of 66 patients with a mean age of $45.4 \pm 9.1$ years were included in the study. PET/CT was performed at the same time in 6 of these patients with suspected urinary system involvement. Only 1 (1.5\%) of 66 patients had a positive urine PCR test. No abnormal genitourinary PET/CT findings were found in any of the patients.

Conclusion: Urine PCR positivity is very rare in patients with COVID-19 infection. In addition, according to our results, it can be said that PET/CT is not an effective imaging method to show COVID-19 urinary system involvement.

Keywords: COVID-19, coronavirus, urine, PCR, PET/CT

\section{Introduction}

Infection factors have caused great morbidity and mortality at different periods in history. Ergotism in $11^{\text {th }}$ century, smallpox in $12^{\text {th }}$ century, leprosy in $1^{\text {th }}$ century, syphilis in $15^{\text {th }}$ century, dysentery in $16^{\text {th }}$ century, tuberculosis in $17^{\text {th }}$ century, typhoid in $18^{\text {th }}$ century, cholera in $19^{\text {th }}$ century, HIV/AIDS in $20^{\text {th }}$ century, but at the beginning of the $21^{\text {st }}$ century, "Severe acute respiratory syndrome (SARS)", caused which is a coronavirus, started to threaten the world (1). About 18 years after that epidemic, a new Coronavirus disease-2019 (COVID-19) outbreak in Wuhan

Address for Correspondence: Gokhan Sonmez, Erciyes University Faculty of Medicine, Department of Urology, Kayseri, Turkey 
spread rapidly to China and then became a global public health problem (2).

In previous reports, it has been reported that COVID-19 can affect not only the respiratory system but also other systems. One of these systems is the urogenital system, and although coronaviruses are basically isolated from the respiratory tract epithelium, it is a matter of curiosity and still controversial whether this virus can be isolated from other body fluids, especially urine $(3,4)$.

Positron emission tomography/computed tomography (PET/CT) is an imaging method used in medicine and often in the diagnosis of oncological diseases. However, it is known that the imaging method used in PET/CT is an indicator not only in oncological conditions but also in infectious and inflammatory conditions (5-7). For this reason, it is important to investigate whether PET/CT, whose use is increasingly widespread with the developing technology, can show organ involvement in coronavirus, in terms of determining new diagnostic methods that can be used in this disease.

In this study, it was aimed to investigate both the isolation of coronavirus from urine and to evaluate the effectiveness of PET/CT in showing urinary organ involvement in COVID-19 patients whose kidneys were affected.

\section{Methods}

\section{Patient Selection and Study Design}

After the ethical approval Ethics Committee of Erciyes University, (approval number: 2020/198 and date: 06.04.2020), patients who were hospitalized in Erciyes University pandemic wards due to COVID-19 infection between June 2020 and December 2020 were included in this prospective study. COVID-19 polymerase chain reaction (PCR) was studied from urine samples of patients between the ages of 18-70 years, who had no active urinary tract infection, and who had a positive COVID-19 PCR test in the throat swab sample in the last 24 hours. The COVID-19 PCR test was positive in the throat swab sample taken within the last 72 hours and the kidney functions [serum creatinine and glomerular filtration rate (GFR)] were normal before, but the renal function deteriorated after COVID-19 (serum creatinine $>2 \mathrm{mg} / \mathrm{dL}$ and/or patients with GFR $<60 \mathrm{~mL} / \mathrm{min} / 1.73$ $\mathrm{m}^{2}$ ) underwent PET/CT. Those with known malignancies, those with previously impaired renal function, known urinary tract stone disease, demonstrated hydronephrosis, those with positive urine culture test, those with known infectious-inflammatory disease (autoimmune, rheumatic, etc.) other than COVID-19, PET/CT withdrawal contraindicated patients (pregnancy, etc.) were excluded from the study. Urine COVID-19 PCR test was also applied to these patients simultaneously.
Previous renal functions of the patients in the PET/CT group were evaluated according to the serum creatinine test performed for another reason and GFR levels in the last 6 months. The results of these tests were obtained from the local hospital registration system or the national patient data registry system (https://enabiz.gov.tr/). Written and verbal informed consent was obtained from all patients included in the study.

\section{Data Collection}

In addition to demographic data of the patients included in the study such as age, gender, body mass index (BMI), additional diseases, history of previous COVID-19, virus positivity rates in urine and renal involvement in PET/ CT were determined and reported.

$\mathrm{PET} / \mathrm{CT}$ images were reviewed and interpreted by the nuclear medicine specialist (A.T.) included in the project team. All urine COVID-19 PCR tests were also performed by the same microbiologists (S.G., O.M.P.).

The data used in the study were obtained from the Erciyes University Hospital Imaging-Automation System and patient follow-up cards created with the data obtained from the patients.

\section{Nucleic Acid Extraction and RT-PCR}

Nucleic acid extraction in urine samples was performed by using EZ1 virus mini kit v2.0 (Qiagen, Hilden, Germany) with automated EZ1 Advanced XL system (Qiagen, Hilden, Germany). Reverse transcriptase-PCR (RT-PCR) method was used for investigation of SARS-CoV-2 RNA in extracted samples. Genesig real-time PCR detection kit for SARS-CoV-2 (Primerdesign Ltd, Chandler's Ford, UK) and Oasig OneStep RT-qPCR master mix (Primerdesign Ltd, Chandler's Ford, UK) were used in accordance with the recommendations of the manufacturer. Positive and negative controls were included in the study and the amplification process was performed on the Rotor-Gene Q (Qiagen, Hilden, Germany) device.

\section{Statistical Analysis}

The distribution characteristics of the data were determined according to the Kolmogorov-Smirnov test and Histogram graphics. Normally distributed numerical data were expressed as mean \pm standard deviation, numerical data not suitable for normal distribution were expressed as median (1-3. Quarter), and categorical data as numbers and percentages. Numerical data of dependent groups showing normal distribution were compared with paired samples t-test. The $p$-value less than 0.05 was considered statistically significant.

\section{Results}

A total of 66 patients were included in the study. PET/ CT was performed in 6 of these patients simultaneously 
with the urine sample. The mean age of the patients included in the study was $45.4 \pm 9.1$ years, and the median BMI was $27.2(24.4-28.8) \mathrm{kg} / \mathrm{m}^{2}$. Thirty-six (54.6\%) of the patients were male and $30(45.4 \%)$ were female. All patients were symptomatic. It was found that 45 (68.2\%) of these patients had COVID-19 lung involvement. While $12(18.2 \%)$ of the patients whose previous data were available had normal renal functions 3-6 months ago (mean creatinine: $0.92 \pm 0.09 \mathrm{mg} / \mathrm{dL}$ ), some impairment in these functions after COVID-19 infection (mean creatinine: $1.32 \pm 0.16 \mathrm{mg} / \mathrm{dL}$ ). Patient characteristics have given in Table 1.

\begin{tabular}{|l|l|}
\hline \multicolumn{2}{|l|}{ Table 1. Patient characteristics } \\
\hline Variable & Value $(\mathbf{n}=66)$ \\
\hline Age (years) & $45.4 \pm 9.1$ \\
\hline Gender (Female/Male) & $30 / 36$ \\
\hline BMI $\left(\mathrm{kg} / \mathrm{m}^{2}\right)$ & $27.2(24.4-28.8)$ \\
\hline Base-line creatinine $(\mathrm{mg} / \mathrm{dL})$ & $0.92 \pm 0.09$ \\
\hline Duration of symptoms (days) & $4.30 \pm 0.65$ \\
\hline Fever $(\mathrm{n}, \%)$ & $24 / 66(36.4 \%)$ \\
\hline BMI: Body mass index & \\
\hline
\end{tabular}

When the urine COVID-19 PCR results of 66 patients were examined, it was seen that only 1 (1.5\%) patient had COVID-19 RNA positivity in the urine. It was found that the only patient with urine PCR positivity was a 69-yearold female patient, with lung involvement in thoracic tomography, and after 7 days of inpatient treatment, the patient was discharged with negativities in the throat swab PCR test and urine PCR test.

While renal functions were normal before, urine COVID-19 PCR test was not positive in any of the 12 patients with impaired renal function after COVID-19. PET/CT was performed in 6 of these patients who met the other inclusion criteria. Two (33.3\%) of six patients were female and $4(66.7 \%)$ were male. The mean age of these patients was $53.2 \pm 3.7$ years. The creatinine values of the patients before and after COVID-19 were $0.78 \pm 0.07$ $\mathrm{mg} / \mathrm{dL}$ and $1.64 \pm 0.26 \mathrm{mg} / \mathrm{dL}$, respectively $(p=0.350)$. The patients' GFR values before and after COVID-19 were $92.66 \pm 5.00 \mathrm{~mL} / \mathrm{min} / 1.73 \mathrm{~m}^{2}$ and $39.33 \pm 6.77 \mathrm{~mL} /$ $\mathrm{min} / 1.73 \mathrm{~m}^{2} \quad(p=0.140)$. No evidence of pathological involvement in the kidneys was found on PET/CT in any of these patients.

\section{Discussion}

The COVID-19 outbreak, which started in 2019 caused by coronaviruses, still continues to be the first agenda item in the world as of 2021 (8). In some previous studies, it has been reported that coronaviruses can be detected in other systems and body fluids (9-13). In addition, it has been shown that the type of coronavirus that causes the COVID-19 epidemic can retain renal epithelial cells and tubular structures (9). In addition, renal involvement has been demonstrated by other histopathological and postmortem studies (14). Based on this information, coronavirus can be expected in the urine samples of some patients. However, today, the isolation of this virus from urine is still controversial. Wang et al. (12) performed PCR test by taking urine samples from 72 patients with positive COVID-19 PCR test in throat swab sample and they did not find COVID-19 positivity in the urine of any patient. Kim et al. (13) reported 2 of 247 urine samples $(0.8 \%)$ and Peng et al. (15) reported in $1(11 \%)$ of 9 urine samples positivity. In a recent review reporting the results of 780 studies and 8,136 specimens analyzed in these studies, it was reported that urine COVID-19 PCR positivity was not found in any patient with a positive nasopharyngeal COVID-19 PCR test (16). Except this, COVID-19 PCR positivity in urine has generally been limited to case reports (11).

In our study, only $1(1.5 \%)$ of 66 patients known to be COVID-19 positive were found to have positive urine. In this context, it can be said that our results are consistent with the literature and the rate of detection of COVID-19 RNA in urine is quite low. In addition, the fact that the only patient with a positive urine PCR test was a patient with mild symptoms, normal renal functions, and clinical improvement in a short time, suggests that there is no correlation between urinary PCR positivity and disease severity.

Since the kidneys are the target of coronaviruses, PET/ $\mathrm{CT}$, which is a highly effective monitor of inflammation, showed kidney coronavirus involvement, which was an expected result for us. In line with this expectation, it has been previously reported that PET/CT may show involvement of other organs in COVID-19 $(17,18)$. Therefore, our study is aimed to be the first study to show the efficacy of PET/CT in coronavirus renal involvement. For this reason, we applied PET/CT imaging to six patients whose renal functions were impaired without any other underlying cause and which we interpreted as COVID-19 kidney involvement. Interestingly, however, pathological involvement was not detected in any renal unit. Therefore, we believe that PET/CT is not an effective imaging method in the patient group we chose.

\section{Study Limitations}

Our study has some important limitations. First, the small number of patients included in the study is the most important limitation for our study. The second important limitation is that histopathological diagnosis methods were not used as an indicator of renal involvement 
in our study due to ethical concerns. Instead, the use of serum creatinine and GFR values, which may vary depending on many factors such as dehydration and drug nephrotoxicity, may have caused partial errors in patient selection. Although various rates have been reported in the literature, up to $20 \%$ of false negatives have been reported in the first PCR test (19). The third limitation of our study is that a single PCR test was applied to urine samples. This may have caused diagnostic errors in the urine samples of some patients.

\section{Conclusion}

According to the results of our study, coronavirus genetic material was not found in the urine in nearly all patients with demonstrated active COVID-19 infection. The same is true for patients with impaired renal function and suspected urinary system involvement. In addition, the results of this study, in which we investigated the efficacy of PET/CT in urinary system involvement for the first time in the literature, showed that PET/CT is not an effective imaging method in urinary system COVID-19 involvement, although it is based on logical explanations.

\section{Authorship Contributions}

Concept: T.D., G.S., A.D., Design: A.D., S.G., A.T., O.Y., Data Collection or Processing: T.D., G.S., E.K., Z.T., Analysis or Interpretation: T.D., G.S., O.M.P., Literature Search: T.D., G.S., S.T.T., Writing: T.D., G.S.

Conflict of Interest: No conflict of interest was declared by the authors.

Financial Disclosure: This study was funded by Erciyes University Scientific Research Projects Coordination Unit (project number: TKB-2020-10238).

\section{References}

1. Burki TK. Pandemics past, present, and future. Lancet Respir Med 2019;7:18-9.

2. Adil MT, Rahman R, Whitelaw $D$, et al. SARS-CoV-2 and the pandemic of COVID-19. Postgrad Med J 2021;97:110-6.

3. Seymen CM. The other side of COVID-19 pandemic: Effects on male fertility. J Med Virol 2021;93:1396-1402.

4. Puelles VG, Lütgehetmann M, Lindenmeyer MT, et al. Multiorgan and Renal Tropism of SARS-CoV-2. N Engl J Med 2020;383:590-2.

5. Arnon-Sheleg E, Israel O, Keidar Z. PET/CT Imaging in Soft Tissue Infection and Inflammation-An Update. Semin Nucl Med 2020;50:35-49.
6. Schönau V, Vogel K, Englbrecht M, et al. The value of ${ }^{18} \mathrm{~F}$-FDG$\mathrm{PET} / \mathrm{CT}$ in identifying the cause of fever of unknown origin (FUO) and inflammation of unknown origin (IUO): data from a prospective study. Ann Rheum Dis 2018;77:70-7.

7. Vaidyanathan S, Patel CN, Scarsbrook AF, Chowdhury FU. FDG $\mathrm{PET} / \mathrm{CT}$ in infection and inflammation-current and emerging clinical applications. Clin Radiol 2015;70:787-800.

8. Asselah T, Durantel D, Pasmant E, Lau G, Schinazi RF. COVID-19: Discovery, diagnostics and drug development. J Hepatol 2021;74:168-84.

9. Martinez-Rojas MA, Vega-Vega O, Bobadilla NA. Is the kidney a target of SARS-CoV-2? Am J Physiol Renal Physiol 2020;318:1454-62.

10. Wang $S$, Zhou $X$, Zhang T, Wang Z. The need for urogenital tract monitoring in COVID-19. Nat Rev Urol 2020;17:314-5.

11. Sun J, Zhu A, Li H, et al. Isolation of infectious SARS-CoV-2 from urine of a COVID-19 patient. Emerg Microbes Infect 2020;9:991-3.

12. Wang $W, X u Y$, Gao R, et al. Detection of SARS-CoV-2 in Different Types of Clinical Specimens. JAMA 2020;323:18434.

13. Kim JM, Kim HM, Lee EJ, et al. Detection and Isolation of SARS-CoV-2 in Serum, Urine, and Stool Specimens of COVID-19 Patients from the Republic of Korea. Osong Public Health Res Perspect 2020;11:112-7.

14. Kudose S, Batal I, Santoriello D, et al. Kidney Biopsy Findings in Patients with COVID-19 . J Am Soc Nephrol 2020;31:195968.

15. Peng L, Liu J, Xu W, et al. SARS-CoV-2 can be detected in urine, blood, anal swabs, and oropharyngeal swabs specimens. J Med Virol 2020;92:1676-80.

16. Bwire GM, Majigo MV, Njiro BJ, Mawazo A. Detection profile of SARS-CoV-2 using RT-PCR in different types of clinical specimens: A systematic review and meta-analysis. J Med Virol 2021;93:719-25.

17. Zou S, Zhu X. FDG PET/CT of COVID-19 . Radiology 2020;296:E118.

18. Sharma P. Incidental COVID-19 Pneumonia on Fluorodeoxyglucose Positron Emission TomographyComputed Tomography: The "New-normal". Indian J Nucl Med 2020;35:276-7.

19. Xiao AT, Tong YX, Zhang S. False negative of RT-PCR and prolonged nucleic acid conversion in COVID-19: Rather than recurrence. J Med Virol 2020;92:1755-6. 